\title{
Research of English Linguistics Classroom Teaching based on Constructivism
}

\author{
Ping Song \\ Dalian Art College, Dalian, Liaoning, 116600
}

\begin{abstract}
Keywords: Constructivism, English Linguistics, Classroom Teaching
\end{abstract}
\begin{abstract}
The English linguistics course is a very important compulsory course for English majors. It mainly studies language development, essence, function and use. This course can help students build a scientific language knowledge system, develop students' scientific awareness and comprehensive language skills. However, at present, the teaching of English linguistics in China still adopts the mode of teacher-based explanation, and the teaching effect is not satisfactory. Therefore, it is necessary to explore an effective teaching mode.
\end{abstract}

\section{Introduction}

With the diversification of foreign language professional training, theoretical, professional, and logical, the linguistics that are considered not to be "practical" have been greatly affected, and the feelings of disgusting learning are widespread. In this case, it is necessary to reconsider the nature, content, training objectives and the significance of the curriculum of the English Linguistics course. Hu Zhuanglin pointed out that our usual promotion of English majors is only satisfied with the training of listening, speaking, reading and writing skills, which makes people feel that there is no big difference between English major teaching and college English teaching. In fact, in addition to the basic stage, the English major focuses on listening, speaking, reading and writing, as well as its own professional courses, including English linguistics. Through the study of this course, students can grasp the basic theoretical knowledge of English linguistics, and have important guiding significance and application value for the actual mastery of language, effective use of language and language work. Therefore, it is very important to strengthen the research on the teaching of English linguistics. This paper aims to construct a diversified English linguistics teaching model based on the constructivism theory and combine the characteristics of English linguistics curriculum, in order to provide new ideas and perspectives for English linguistics teaching practice.

\section{Constructivist theory}

Constructivist teaching theory is an important branch of cognitive learning theory. It was first proposed by the famous Swiss psychologist Piaget in the 1930s, and later by many psychologists and educators in Ausubel and Bruner. Under the unremitting efforts, this theory has been enriched and developed and gradually formed a relatively complete system. Constructivism believes that learning is a dynamic process of dynamic construction. It is the learner himself. With the help of teachers and learning partners, in the real situation, in the form of collaborative conversation, consciously and actively construct the meaning of knowledge, while learning The cognitive structure of the person is also reconstructed. In order to help learners to actively and effectively complete the construction of the knowledge they have learned, the creation of learning situations with real problems, rich information resources and the provision of collaborative learning models become indispensable conditions. Constructivism emphasizes the important role of context in the construction of meaning, and regards the creation situation as a necessary premise of meaning construction. It even thinks that the influence of environment on learning exceeds the content of learning, and that learning is always associated with certain situations. Constructivism The envisioned learning context should be a scenario that facilitates learners to engage in discovery and exploratory learning. Constructivism also emphasizes the key role of collaborative learning in the construction of meaning. It is believed that the construction of meaning occurs in the conversation 
with others and is the result of social interaction. Due to the different experience of each person, there are also individual differences in cognitive ability. The interpretation of the same input may not be consistent, but through the cooperation and communication with others, the constructed knowledge system will be more perfect. The core of Ausubel's assimilation theory is: whether students can acquire new information depends mainly on the relevant concepts already in their cognitive structure. Meaning learning is to interact with existing concepts in the cognitive structure of students through new information. It leads to the assimilation of the meaning of new and old knowledge, which not only makes the new knowledge gain meaning, but also makes the old knowledge modified to obtain new meaning. Bruner believes that in the teaching process, the student is an active explorer. The role of the teacher is to create a situation in which students can explore independently, rather than providing ready-made knowledge. Constructivist teaching creates more opportunities for students to manage themselves than traditional teaching, requiring students to complete tasks in complex real-world situations. In addition, constructivism also places great emphasis on the social interaction between teachers and students, students and students. They believe that through cooperation and discussion, students can see all aspects of things. There are many studies on the application of constructivism theory to teaching practice at home and abroad, but most of them are in the fields of mathematics and biology, but very little in foreign language teaching. In this few studies, it is mainly about constructivism and English electrification and network teaching.

\section{Construction mode in linguistic teaching}

In order to highlight the student's subjective status, teachers should combine the content of the course, design activities, create opportunities to stimulate students' active participation in the construction of the consciousness, and change the situation in which the teacher sang "one-man show" and the students passively listened to. Because the linguistics course involves many terminology and linguistic theories, this knowledge is relatively unfamiliar and abstract for students. If students actively participate in their own activities and turn their knowledge into their own autonomous behaviors, then Achieve meaningful knowledge building. For example, English morphology is about the internal structure of words and the study of word formation, which is very helpful for students' English learning. After teaching this chapter, assigning group tasks to students to discuss how morphological learning can help English learning can be summarized into meaningful vocabulary memory methods. In the classroom, each group reports its own discussion results through examples. Through their own discussions and feedback from others, students will form their own understanding of the importance of morphology, and can form a network of previously vocabulary knowledge and improve the network. The efficiency of remembering words. In the process of finding data discussion, students can actively think about, truly exert their potential and build a knowledge structure.

Constructivism theory holds that teachers are the leaders and instructors of meaning construction. Teachers should introduce appropriate linguistic learning methods according to students' cognitive characteristics in teaching, and cultivate students' thinking ability and problem-solving ability. For a long time, teachers have used content and results as the core of teaching in teaching, ignoring the cultivation of learning methods and learning strategies. Therefore, the thinking structure of most students is not suitable for constructive teaching, and there is a lack of critical thinking required for linguistic teaching. Systematic thinking and divergent thinking. Therefore, in addition to explaining the basic theories, teachers can also guide students to understand the research methods of linguistics and integrate the research concepts and methods into the classroom. For example, in the introductory class, students should be introduced to some linguistic learning strategies. For example, the study of this course should start from the whole, first macroscopically and then microscopically, and do "see both forest and wood". There are many branches of linguistics, which are divided according to pronunciation, structure and meaning. The arrangement of each chapter is also divided according to these three elements. Teachers should instruct students to first understand the content of the course at the macro level. Read the catalog carefully, because the catalog is a high-level 
summary of the author's content. It not only contains the main content of linguistics, but also clearly reflects the links between the chapters. On the basis of grasping the content of the textbook as a whole, we will enter micro-learning. There are many concepts and terminology in linguistics. Students should be guided by understanding, and they should pay attention to understanding and examples. Every time you touch a new term, first understand its definition, then look at the examples given, and combine the examples to abstract the concept of abstraction, easy to understand and remember. For the difference between different concepts, a comparison and comparison method can also be adopted. By comparison, we can find similarities between different concepts, such as phonology and morphology. From the morphological point of view, the suffix is the same. "The root of the word is about the sound, and the other is about the vocabulary, so that students can easily remember these two terms, which can better understand the meaning of the term."

Many students are bored and afraid of the linguistics course. After the course, the mastery of knowledge is not very strong. The reason is that the course is abstract, theoretical, and has many concepts. The second is the students' The importance of understanding is not enough. Lack of interest in learning the course. Only by cultivating students' interest in learning linguistics will they have the motivation to learn. First, the teacher should explain to students the importance of linguistics in the entire discipline and the relationship with other courses. For example, linguistic knowledge can help students analyze literary works, and linguistic knowledge can be better when translating. The effects, etc., to stimulate students' interest in learning linguistics courses. At the same time, teachers can also link examples in everyday life with boring linguistic theories to let students understand the practical value of linguistic theories. When talking about the rhetorical effect of the same word, you can use some advertising terms as an example, such as Yuba's advertising word "Bath in the heart." When speaking about dialects, you can ask classmates from different places to talk about dialects in their hometowns, compare different vocabulary and usage; when speaking English idioms, encourage students to compare Chinese idioms and find out the similarities and differences. Through the examples around, students can not only remember linguistic knowledge, but also stimulate their interest to find the language phenomenon around them.

The constructivist view of learning believes that learning is the result of interaction between teachers and students. Therefore, research activities should be carried out in classroom teaching. Students should participate in the construction of knowledge. They should cultivate their rational thinking and scientific consciousness on the basis of mastering theoretical knowledge. Compared with other liberal arts college students, English majors have a significantly higher discerning ability than other liberal arts college students, but this gap has gradually narrowed with the increase of grades. The curriculum of senior English majors is mainly based on knowledge courses. As a main course, linguistics courses should pay attention to cultivating students' thinking ability, which is also advocated by constructivist learning theory. First of all, we must cultivate students' interdisciplinary awareness and broaden their thinking skills. Linguistics itself is an interdisciplinary science that studies both linguistic and natural science methods, such as statistical and experimental methods, corpus research methods, and inductive and deductive logic., analysis, synthesis and comparison and other research methods. Linguistics is also closely related to other disciplines such as psychology, neuroscience, translation, and foreign language teaching. Second, students should be encouraged to think positively and motivate them to think and solve problems. For example, teachers should encourage students to explore the relationship between language and thinking, the relationship between language and culture, the relationship between language and society, etc., and encourage students to compare the discussion of these issues with different linguists. Correctly understand the relationship between language and culture, language and society, and improve their ability to think critically. In addition to the training of students' thinking ability, the teachers themselves must have a strong sense of speculation, constantly change their teaching philosophy and teaching methods, and put the students' thinking ability training in the first place. 


\section{Conclusion}

The constructivist teaching concept provides a new model for classroom teaching, and explains the new concept of learning for students who are accustomed to passive acceptance, thus bringing new enlightenment and experience to the teaching and learning of English linguistics classroom. In the English linguistic classroom teaching, teachers should pay special attention to the following five aspects: strengthen teaching interaction, pay attention to evaluation effect, teach learning strategies, guide students to learn independently and improve teaching methods. These aspects help students build knowledge and build abilities.

\section{References}

[1] Jiang Hui, Sun Yongjun. Reflections on the Orientation and Teaching Mode of College English Major Linguistics Courses [J]. Journal of Liaoning Normal University (Social Science Edition) 2010(3) : 123 - 124.

[2] Cheng Xiaoguang. The philosophical foundation of social constructivism [J]. Foreign Language and Foreign Language Teaching, 2005(1): 3 - 7.

[3] Fan Lin. The Correspondence between Constructivism Teaching Theory and English Teaching Reform [J]. Foreign Language and Foreign Language Teaching, 2003(4): 28 - 32.

[4] Huang Guowen. From results-centered to process-centric [J]. Foreign Language and Foreign Language Teaching, 2000(10): 1, 32.

[5] Yan Yumei. Thoughts on the Teaching of English Majors in Linguistics [J]. Foreign Language and Foreign Language Teaching, 2007(8): 31 - 33, 50. 